\title{
Perspectives of the ALICE Experiment
}

\author{
Paolo Giubellino \\ INFN Torino, 1 VIa Pietro Giuria, I-10125 Torino, Italia
}

Received on 14 August, 2003.

\begin{abstract}
The Large Hadron Collider, now under contruction at the European Center for Nuclear Research, represents a unique opportunity for Heavy-Ion Physics. It will provide nuclear collisions at a center-of-mass energy 30 times higher then the present Relativistic Heavy Ion Collider at BNL, currelty the highest energy nuclear accelerator. The LHC will open for this field a new era, in which particle production will be dominated by hard processes, and the energy densities will possibly be high enough to treat the generated quark-gluon plasma as an ideal gas. While RHIC is providing a wealth of interesting data, many physicists are working hard to prepare the experiments which will run at the LHC. ALICE, A Large Ion Collider Experiment, the dedicated detector designed to study nucleus-nucleus collisions at the LHC, is developing rapidly: the R\&D is essentially complete, and large parts of the main detectors are in production. The scientific motivations and present status of Heavy Ion Physics, can be found in the review by T. Kodama [1]. In the following, I will summarize the experimental conditions at the LHC with nuclear beams, describe the main detector components of ALICE and briefly discuss the physics program of the experiment.
\end{abstract}

\section{Introduction}

The LHC is now scheduled to start operation in 2007. The acceleration of nuclear beams is part of the initial program, and a Heavy Ion pilot run is foreseen for the first LHC year. The LHC will dedicate every year few weeks of running to Heavy Ions. The LHC will provide $\mathrm{Pb}$ ions at a center-ofmass energy of $5.5 \mathrm{TeV}$ per nucleon pair, which represents a jump of more than an order of magnitude with respect to the RHIC energy. It will therefore lead into a radically new energy region, previously reached only in the interactions of the highest energy cosmic rays. Four experiments will operate at the LHC. One of these, ALICE, is dedicated to Heavy Ions, another one, CMS, is dedicated primarily to pp but features a well defined HI program, while a third, ATLAS, has expressed an interest in running with Heavy Ions. Being the only dedicated experiment for the study of nuclear collisions at the LHC, ALICE is essentially a "Heavy Ion Program" which covers in one experiment the full range of relevant observables. CMS, on the other hand, is optimized for the study of high transverse momentum processes, and will therefore focus on these observables only.

The higher energy will improve by large factors, typically by an order of magnitude, all parameters relevant to the formation of the Quark-Gluon-Plasma: energy density, size and lifetime of the system. The initial temperatures will largely exceed the calculated critical temperature for QGP formation, therefore allowing the study of QGP in its asymptotic ideal gas form. In the central region the net baryon number density will essentially vanish, improving the ease of comparison with lattice QCD calculations and the closeness to the conditions of the primordial universe. The region of $x_{B j}$ explored will extend singificantly towards smaller- $x$ values, while particle production will be dominated by hard rather than soft processes. Moreover, it will be possibile to exploit a wider set of relevant observables as compared to previous accelerators, thus substantially enhancing the understanding of the properties of the system. First of all, the higher energy and the high luminosity will improve access to hard probes sensitive to the nature of the medium at the earliest stages. The study of jet production and therefore of the propagation of fast partons will find at the LHC its ideal environment, allowing the study of jet fragmentation functions up to well over $100 \mathrm{GeV} / \mathrm{c}$ of jet $E_{\mathrm{t}}$. The excellent PID capability and wide coverage in transverse momentum of ALICE, which has good resolution from below $100 \mathrm{MeV} / \mathrm{c}$ to $100 \mathrm{GeV} / \mathrm{c}$, will allow the detailed study of the fragmentation functions. The study of the jet recoiling against a photon will allow the measurement of the fast parton energy loss. The study of the heavy quark potential will benefit from the possibility to measure both the charmonium and bottomium families, which provide a wide range of radii and binding energies, and of the possibility of measuring in the same experiment the production of open charm and beauty mesons, and even of the contribution of B meson decays to the $\mathrm{J} / \psi$ yield. The temperature of the medium should be high enough to allow a precise direct measurement of the prompt photon spectrum, which would be a direct thermometer of the system.

In addition to the new wealth of information provided by the hard probes, the very high multiplicity, several thousands of charged partciles per unit $\eta$, will allow the measurement of a large number of observables on an event-by-event basis: impact parameter, multiplicity, particle composition and spectra and HBT parameters of the system. Therefore single event analysis, and in particular the study of non-statistical fluctuations associated to critical phenomena, can be effectively performed at the LHC. 


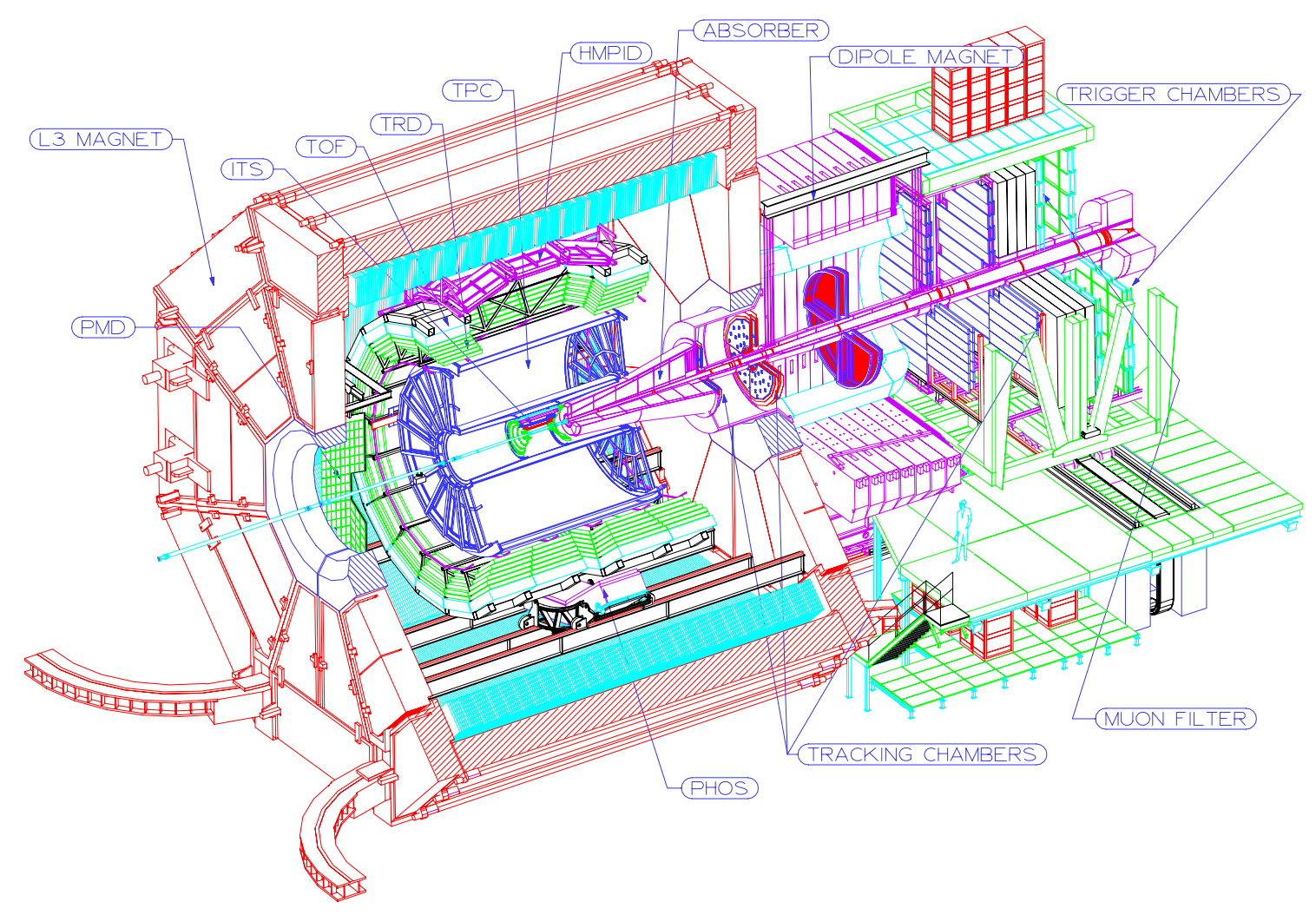

Longitudinal view of the ALICE detector.

The present ALICE design [2-5] is a multi-purpose one, addressing in a comprehensive all relevant observables, and foresees the measurement and identification of most of the produced hadrons, leptons and photons. A Longitudinal view of the ALICE detector is shown in fig. . The detection of muons is performed by a dedicated spectrometer, with a very large warm dipole magnet, in the forward region $(2.4 \leq \eta \leq 4)$. Hadrons, electrons and photons are measured in the central region $(-0.9 \leq \eta \leq 0.9)$ by a complex system relying on very high-granularity, yet relatively slow drift detectors, a weak, very-large volume magnetic field, and on specially developed detectors for particle identification. Tracking is performed combining the information from a large TPC, which is the main tracking detector, a system of six barrel layers of silicon detecotrs, providing high-resolution tracking and precise vertex information, and a Transition Radiation Detector. For Particle identification, in addition to the measurement of the specific energy loss in the tracking detectors, which provides excellent separation at low momenta and some identification capability in the relativisitc rise region, a dedicated high-resolution TOF barrel provides the identification for the bulk of the intermediatemomentum particles, while a Ring-Imaging Cerenkov extends the particle identification to higher momenta in a reduced acceptance region. Electrons are identified using the TRD information. Short-lived particles are identified by exploiting the excellent tracking and vertexing capabilities of the experiment. Photons are measured in a high-granularity crystal calorimeter of limited acceptance. The event charac- terization is completed by the measurement of the charged multiplicity over a wide acceptance $(-5.1 \leq \eta \leq 3.4)$, of the forward photon multiplicity $(-3.5 \leq \eta \leq-2.5)$ and of the very-forward energy flow, measuring spectators in Heavy-Ion collisions and thus the impact paremeter. A double array of scintillation counters (V0) is used as the main interaction trigger, while a double array of quartz Cherenkov counters provides the precise time of the event.

\section{ALICE overview}

The ALICE rapidity acceptance has been chosen to be large enough to allow the study of particle ratios, $p_{\mathrm{t}}$ spectra and HBT (Hanbury-Brown-Twiss) radii on an event-by-event basis, meaning several thousand reconstructed particles per event. Detecting the decay products of particles at $p_{\mathrm{t}}<m$ requires about 2 units in rapidity (for masses above 1-2 $\mathrm{GeV} / \mathrm{c}^{2}$ ) and a corresponding coverage in azimuth. A similar acceptance is necessary to collect a reasonable (few $10^{3}$ ) sample of $\Upsilon$ in the dielectron channel, given the expected luminosity and running time. Again a similar value of acceptance is required to collect sufficient statistics to measure hadronic decays of charmed mesons with good significance. The coverage of the central detector $(|\eta|<0.9$, full $2 \pi$ in $\phi$ ) has therefore been chosen as a compromise between acceptance requirements and cost.

ALICE has been specifically designed to maximize momentum coverage, from $\approx 100 \mathrm{MeV} / c$, the lowest values 
relevant for thermodynamical studies, to $\approx 100 \mathrm{GeV} / c$, the transverse momentum of the leading particles of jets with transverse energy well over $100 \mathrm{GeV}$. The measurement of numerous precision points over a long measured track length in a moderate magnetic field and with minimal material allows to satisfy both requirements.

Tracking The design of the ALICE tracking system has primarily been driven by the requirement for safe and robust track finding. It uses mostly three-dimensional hit information and dense tracking with many points in a moderate magnetic field.

The Time Projection Chamber is the main tracking detector. It provides track finding, momentum measurement and particle identification via $\mathrm{d} E / \mathrm{d} x$. With its $88 \mathrm{~m}^{3}$, it is the largest TPC ever built. The TPC has an inner radius of $90 \mathrm{~cm}$, given by the maximum acceptable hit density $\left(0.1 \mathrm{~cm}^{-2}\right)$, and an outer radius of $250 \mathrm{~cm}$, given by the length required for a $\mathrm{d} E / \mathrm{d} x$ resolution of $<10 \%$, necessary for particle identification. The 72 pad-readout chambers feature 570,000 channels.

The inner tracker consists of six cylindrical layers are located at $\mathrm{r}=4,7,15,24,39$ and $44 \mathrm{~cm}$. Four layers will have analog readout to provide particle identification via $\mathrm{d} E / \mathrm{d} x$ in the $1 / \beta^{2}$ region, giving the ITS a stand-alone capability as low- $p_{\mathrm{t}}$ particle spectrometer. Because of the particle density and to achieve an impact parameter resolution below $100 \mu \mathrm{m}$, pixel detectors have been chosen for the innermost two layers, and silicon drift detectors for the following two. Double-sided silicon micro-strip detectors equip the two outer layers. The minimization of the material thickness being absolute priority, the average thickness, all included, is kept below $1 \%$ of $X_{0}$ per layer.

The overall performance of the ALICE tracking system has been simulated taking into account the details of the detector performance.

At target multiplicity the tracking efficiency in the TPC is better than $90 \%$ practically independent of $p_{\mathrm{t}}$ down to about $100 \mathrm{MeV} / \mathrm{c}$. For lower multiplicities, the efficiency would rapidly improve, reaching values $\approx 97 \%$ below 4000 charged particles per unit of rapidity. For very low transverse momenta the ITS has the capability to function as a stand-alone tracker, providing tracking and PID down to below $100 \mathrm{MeV} / \mathrm{c}$. This feature is particularly important to reconstruct the low- $p_{\mathrm{t}}$ decay products of Hyperons.

The momentum resolution, including ITS, TPC and TRD, is better than $\approx 1.5 \%$ for momenta between 200 $\mathrm{MeV} / \mathrm{c}$ and $2 \mathrm{GeV} / \mathrm{c}$, and it is still close to $\approx 12 \%$ at 100 $\mathrm{GeV} / \mathrm{c}$. The angular resolution is on average $\approx 0.5 \mathrm{mrad}$.

These momentum and angular resolutions, coupled with the excellent capability to disentangle close tracks, provide ALICE with the capability of measuring with good precision the HBT parameters for a source of radius well in excess of $10 \mathrm{fm}$.

The impact parameter resolution improves rapidly with transverse momentum, and it reaches $60 \mu \mathrm{m}$ at $1 \mathrm{GeV} / \mathrm{c}$. This value is adequate for efficient identification of the short-lived B and D mesons, which have $c \tau$ of the order of 100 to $300 \mu \mathrm{m}$.

Particle Identification One of the distinctive features of ALICE is the particle identification capability, which is realized using a number of different techniques. This robust PID system allows the identification of a large number of protons, pions and kaons in each $\mathrm{Pb}-\mathrm{Pb}$ event, so that their transverse momentum spectra can be studied on an eventby-event basis. Thanks also to the good momentum and angular resolution, the decay $\phi \rightarrow \mathrm{K} \mathrm{K}$ can be measured with great precision, with a mass resolution of about $2 \mathrm{MeV} / \mathrm{c}^{2}$. Coupled with the excellent vertexing capability of the ALICE tracking system, Hyperons (including the rare $\Omega$ ) and the hadronic decays of charmed mesons can be measured. The measurement of hadronic decays of charmed mesons is an ideal example of the combined power of the ALICE detector systems, using PID, tracking and vertexing. The result is a measurement of the neutral $\mathrm{D}$ meson decaying into $\mathrm{K} \pi$ with a significance of about 37 , which allows a direct study of the $p_{\mathrm{t}}$ dependence of charmed meson production down to $1 \mathrm{GeV} / \mathrm{c}$ of $p_{\mathrm{t}}$ (significance $\approx 12$ ).

Hadron identification is provided over a large part of the phase space by the combination of $\mathrm{d} E / \mathrm{d} x$ measurement in the silicon and in the TPC with a high-resolution $(<100 \mathrm{ps})$ barrel TOF at $\mathrm{r}=3.7 \mathrm{~m}$. The $\mathrm{d} E / \mathrm{d} x$ resolution of the TPC is sufficient to provide good $\pi$ identification in the relativistic rise region, thus providing an additional handle for the study of hard probes. The TOF uses innovative multigap resistive plate chambers (MRPC), featuring 160,000 precision readout channels.

A Ring Imaging Cherenkov detector of the proximity focusing type, using a liquid $\mathrm{C}_{6} \mathrm{~F}_{14}$ radiator, covering about $15 \%$ of the acceptance of the ALICE central detectors, allows the identification of hadrons up to higher momenta ( $\pi / K$ to $3 \mathrm{GeV} / \mathrm{c}$ and $K / \mathrm{p}$ to $5 \mathrm{GeV} / \mathrm{c}$ ) .

Leptons Both electrons and muons are measured in ALICE, the electrons in the central barrel and the muons in a dedicated forward arm. The acceptance and resolution are such as to allow the measurement of heavy quarkonia families down to $p_{\mathrm{t}} \approx 0$ and with good separation of the different "onium" states for both $e e$ and $\mu \mu$ channels. The electron measurement coupled with the vertexing capability allows the direct measurement of the yield of B and D mesons via their semileptonic decay, thus providing the natural reference for the "onium" measurements.

Electrons are identified in ALICE by combining the particle identification capability of ITS, TPC and TOF and complementing it with a dedicated Transition Radiation Detector. The TRD consists of six layers of radiator followed by Time Expansion Chambers filled with Xenon/ $\mathrm{CO}_{2}$, providing an $\mathrm{e} / \pi$ rejection power of 100 when operating in a high multiplicity environment. The TRD fast tracking capability is used to trigger on high- $\mathrm{p}_{\mathrm{t}}$ leptons, thus enriching their statistical sample, and hadrons, thus providing an essential 
trigger for jet leading particles. The detector in its full configuration covers the central barrel region of ALICE, and consists of 540 modules organized in 36 azimuthal sectors, corresponding to the azimuthal segmentation of the TPC. The TRD needs therefore $800 \mathrm{~m}^{2}$ of high-granularity readout chambers, equipped with over one million electronics channels.

The forward muon spectrometer allows the study of vector meson resonances via their $\mu^{+} \mu^{-}$decay. The signals appear on a continuum due to $\mathrm{B}$ and $\mathrm{D}$ meson decays and Drell-Yan processes. The spectrometer has an efficiency for dimuons better than $90 \%$ and a mass resolution better than $100 \mathrm{MeV} / c^{2}$ in the $\Upsilon$ region and better than $70 \mathrm{MeV} / c^{2}$ in the $J / \Psi$ region. The momentum precision is about $1 \%$. The muon spectrometer consists of a composite absorber $\left(\approx 10 \lambda_{I N T}\right)$ starting close to the interaction point (one meter) to reduce the $\mu$ background due to $\pi$ and $K$ decays. A high-density small angle absorber with a central hole shields the spectrometer from the particles emitted at angles from 0 to 2 degrees and allows the LHC beams to traverse the spectrometer. At the end of the spectrometer is located a final muon identifier filter wall, consisting $\approx 10 \lambda_{I N T}$ of iron. The spectrometer magnet is a large warm dipole magnet with a field of $0.7 \mathrm{~T}$, giving a $3 \mathrm{Tm}$ field integral. The dipole accepts muons at angles smaller than 9 degrees. Ten planes of thin multiwire proportional chambers with cathode pad readout placed in front, inside and following the dipole measure the muon trajectories. Two single gap RPC planes detect the particles emerging from the muon filter and provide the trigger for the spectrometer.

Photons Prompt photons, $\pi^{0}$ 's and $\eta$ 's are measured in a single-arm, high-resolution electromagnetic calorimeter. The accuracy of the single inclusive photon spectra will be determined by the systematic errors on photonreconstruction efficiency and by the knowledge of the decay background. An acceptable systematic error can be obtained only at low channel occupancy and therefore requires a calorimeter with small Molière radius, $R_{\mathrm{M}}$, at a large distance $(\approx 5 \mathrm{~m})$ from the vertex. The acceptance has been defined such as to keep the statistical errors below the expected systematic ones. The PHOS is located $5 \mathrm{~m}$ vertically beneath the interaction region and is built from $\mathrm{PbWO}_{4}$, a material with small Molière radius and high light output.

Jets Jets are measured in ALICE by reconstructing charged particles in a cone around a "seed" particle of high $p_{\mathrm{t}}$. The TRD triggering capability provides the possiblity to record $\approx 10^{6}$ events per standard $\mathrm{Pb}$ run with jet energy above $100 \mathrm{GeV}\left(10^{4}\right.$ above $\left.200 \mathrm{GeV}\right)$. Therefore ALICE will collect sufficient statistics to exploit its excellent tracking and PID capability for the study of detailed jet fragmentation functions, which is a very sensitive tool for the study of jet quenching. Using the photon measured in the PHOS as a tag, ALICE can study photon-jet back-to-back pairs. In this way, the average jet energy is defined by the photon measurement, and the fast parton energy loss is directly accessible. The range of jet energies accessible with such a measurement would be greatly enhanced by the proposed large acceptance electromagnetic calorimeter. If available, the EMCal would also significantly improve the measurement of the jet energy, by complementing the measurement of the charged particles performed by the tracking system, and provide an improved jet trigger.

\section{References}

[1] T. Kodama, Brz. J. Phys. 34, 206 (2004).

[2] ALICE Technical Proposal N. Ahmad et al., CERN/LHCC/95-71 (1995).

[3] ALICE TP addendum, S. Beolé et al., CERN/LHCC/96-32

[4] ALICE TP addendum, G. Dellacasa et al., CERN/LHCC/9913

[5] P. Giubellino, Eur.Phys.J.direct C4S1:05, 2002

[6] Alice Collaboration, Physics Performance Report, in preparation

[7] Alice collaboration, ALICE Physics, Theoretical Overview, ALICE-INT 2002-025 